\title{
A Singular Example for the Averaged Mean Curvature Flow
}

\author{
Uwe F. Mayer
}

\begin{abstract}
An embedded curve is presented which under numerical simulation of the averaged mean curvature flow develops first a loss of embeddedness and then a singularity where the curvature becomes infinite, all in finite time. This leads to the conjecture that not all smooth embedded curves persist for all times under the averaged mean curvature flow.
\end{abstract}

Key words. Averaged mean curvature flow, loss of embeddedness, immersed curve, blowup of curvature, numerical experiments

AMS subject classification 2000: 35R35, 58G11, 31C20, 65M99.

\section{INTRODUCTION}

We describe numerical experiments in two space dimensions for a well-established geometric evolution law, the averaged mean curvature flow, also known as the area-preserving mean curvature flow, or, in higher dimensions, as the volume-preserving mean curvature flow. The key example presented in this paper is an embedded curve that develops first a self-intersection and then a singularity of blowup type for the curvature, all in finite time.

It had been generally believed that this type of flow will keep embedded curves smooth, so this example comes as a surprise. Of course, it has been conjectured for quite some time that the averaged mean curvature flow can drive curves to a loss of embeddedness; such a conjecture enters the literature with [Gage 1986]. This has recently analytically been proved to be correct [Mayer and Simonett 2000]. However, the general belief remained that no essential singularity will occur, and has been founded on the behavior of the nonaveraged mean curvature flow. The pioneering work of Gage and Hamilton [1986] and of Grayson [1987] showed that the nonaveraged mean curvature flow, if rescaled homothetically to preserve the enclosed area, will evolve any embedded curve into a circle.

Let $\Gamma_{0}$ be a closed embedded curve in $\mathbb{R}^{2}$. The averaged mean curvature flow is governed by

$$
V(t)=-(\kappa(t)-\bar{\kappa}(t)), \quad \Gamma(0)=\Gamma_{0},
$$

where $\bar{\kappa}(t):=|\Gamma(t)|^{-1} \int_{\Gamma(t)} \kappa(t) d \sigma$ denotes the average of the curvature. Here $\Gamma=\{\Gamma(t): t \geq 0\}$ is a family of smooth immersed orientable curves, $V(t)$ denotes the velocity of $\Gamma$ in the normal direction at time $t$, while $\kappa(t)$ stands for the curvature function of $\Gamma(t)$. The name mean curvature flow comes from the higher-dimensional model.

The averaged mean curvature flow evolves curves in such a way that the length decreases. Also, if $\Gamma$ is embedded the flow preserves the area of the region $\Omega(t)$ enclosed by $\Gamma(t)$ [Huisken 1987; Escher and Simonett 1998]; this explains the alternative name of this flow, "area-preserving" mean curvature flow. Note that the evolution law (1.1) does not depend on the local choice of the orientation.

The example studied here is similar to, but not identical with, the one proposed in [Gage 1986], where it was suggested as an example which should exhibit a loss of embeddedness under the averaged mean curvature flow. A curve modeled on the one proposed by Gage was also numerically investigated in [Mayer 
and Simonett 2000], but the curve in the latter paper remains regular due to a slight variation of parameters as compared to the curve studied here. This will be explained in more detail below.

The assumption of embeddedness constitutes the novelty of this example. It is general mathematical folklore - based on numerical simulations - that for the averaged mean curvature flow certain immersed curves, such as a figure-eight or a limacon, will develop a singularity, even though no analytical proof is known. But all previously proposed singularity-producing immersed curves have a turning number different from one, while any curve arising from an initially embedded curve must necessarily have a turning number equal to one. Also, this lack of analytical results is in contrast to the nonaveraged mean curvature flow, where Angenent gave detailed results on the nature of the singularity that occurs for a lima con [Angenent 1991].

The numerical experiments have been performed using two independent implementations of the averaged mean curvature flow. The first uses a numerical scheme developed by the author. It is based on the gradientflow structure of certain free boundary problems, of which the averaged mean curvature flow is the special case of the $L_{0}^{2}$-gradient, the index 0 stands for functions of zero average; see [Mayer 2000]. The second implementation is the multi-model Surface Evolver developed by Brakke [1992], also based on a gradient approach. In closing of this introduction we formally state the conjecture obtained from the numerical experiments.

Conjecture There are smooth embedded curves in $\mathbb{R}^{2}$ which, under the averaged mean curvature flow, develop in finite time a singularity where the curvature becomes infinite.

\section{THE EXPERIMENTS}

\section{A The Example}

The images on these two pages are based on computations with the algorithm presented in [Mayer 2000]. The time step for this semi-implicit method was $10^{-7}$, with a lower spatial threshold of $10^{-4}$. The initial curve had about 1400 points. Every 100 iteration steps a check was performed whether two neighboring points on the curve were closer than the spatial threshold, and if so, then one of them was removed. Every 500 iteration steps each point was replaced with a point equidistant to the current two closest neighbors. The replacement point was chosen on a circular arc through the two neighbors, so as to preserve the numeric curvature. As already mentioned, the computations were confirmed with the Evolver [Brakke 1992], to minimize the chance that the results are a numerical fluke of the particular algorithm. Of course, the parameters at which the singularity occurred were slightly different with the different numerical methods, but the phenomenon occurred just the same.
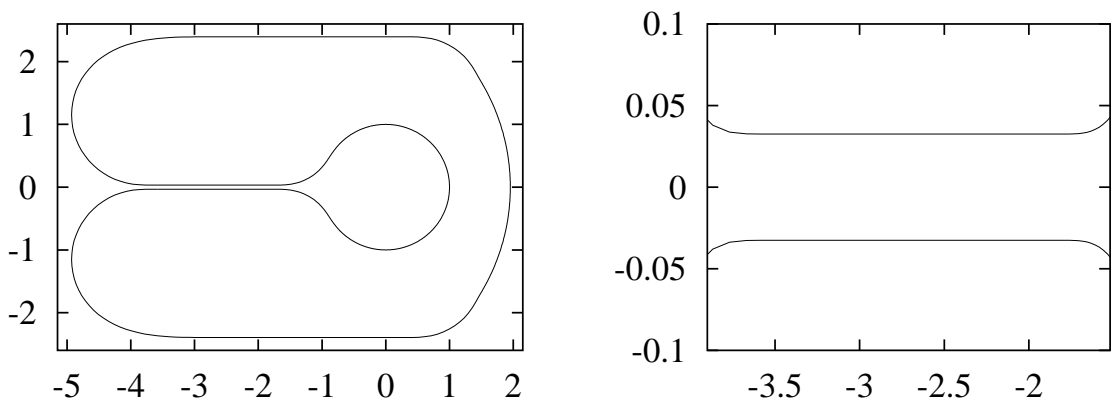

Fig. 1 Left: the initial curve; the two parallel segments in the center of the curve are about 0.065 units apart. Right: close-up of the slit; the vertical scale has been magnified about 10 times more than the horizontal one. 

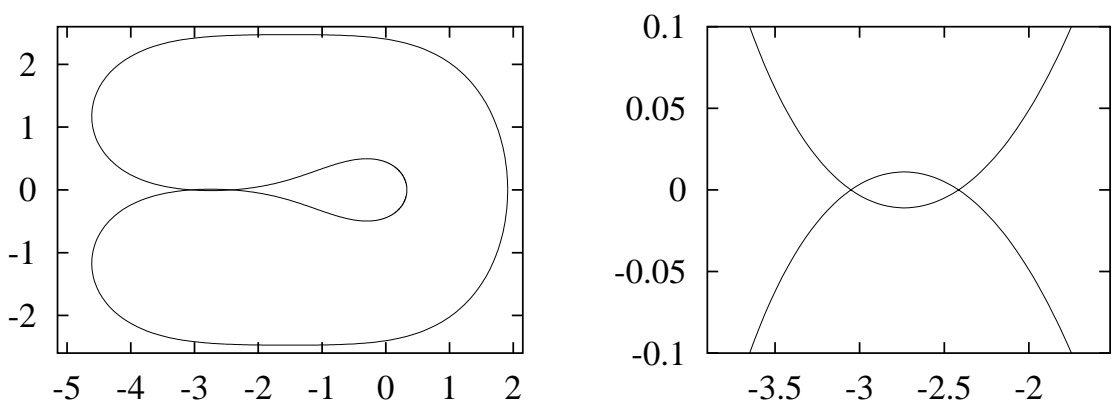

Fig. 2 Left: the curve at time $t=0.4$; the two parallel segments in the center of the initial curve have created an overlap. Right: close-up of the overlap.
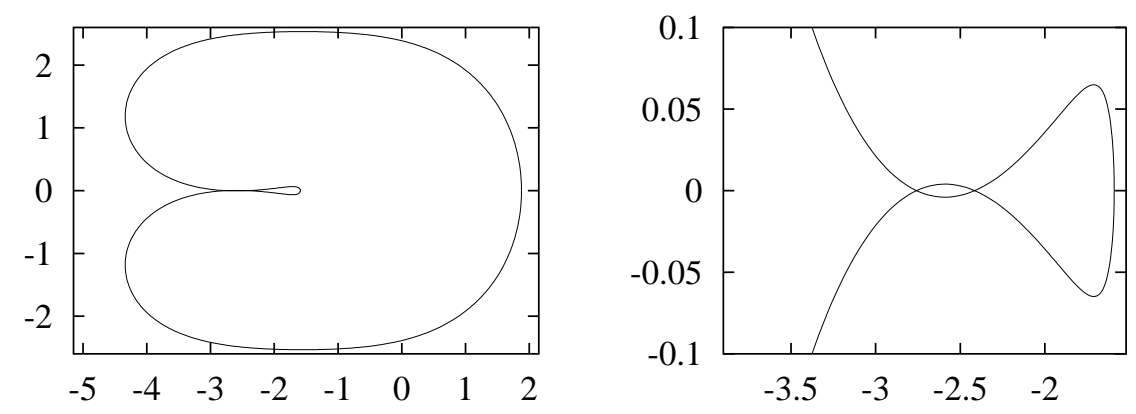

Fig. 3 Left: The curve at time $t=0.73$; the center loop is fairly tight already. Right: close-up of the center.
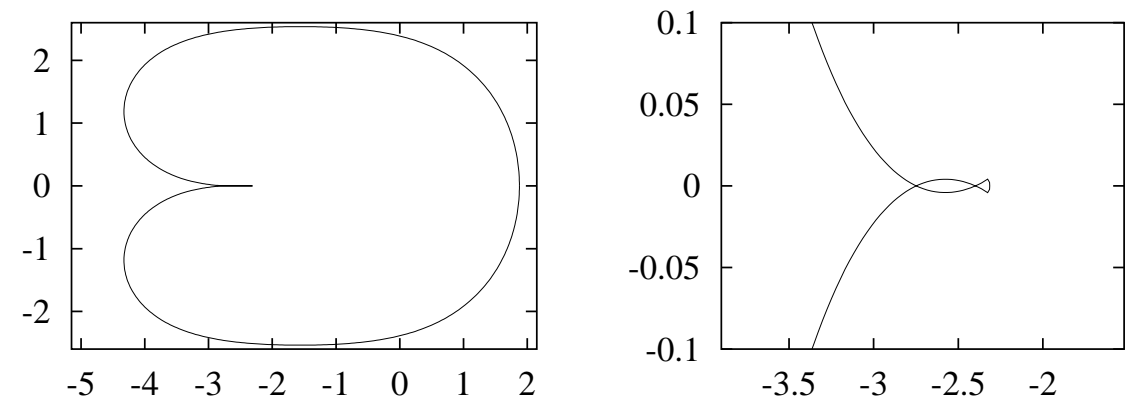

Fig. 4 Left: The curve at time $t=0.746$; the center appears to be already singular. Right: close-up of the center; the small loop will close down before the overlap has time to pull apart, and the curvature will blow up (become infinite). 
One motivation for looking at this example was the work by Angenent [1991], in which the effect of the nonaveraged mean curvature flow on a limacon is studied. The small loop of the lima con is reminiscent of the small loop occurring in the curve depicted above. By the theorem of turning tangents for a simple closed curve $\Gamma$ one has the formula

$$
\bar{\kappa}=\frac{1}{L} \int_{\Gamma} \kappa d s=\frac{2 \pi}{L},
$$

where $L$ is the length of $\Gamma$. It follows that if $L$ is bounded from below and if $\kappa$ is big, then the normal velocity $V=-\kappa+\bar{\kappa}$ ought to be dominated by $\kappa$ alone. This is roughly the case in the center of the curve, and so it ought to behave similarly to the limacon. On the other hand we do not have quite this scenario, because the curvature in the example has a sign change in the small loop, hence the curvature is not big everywhere in the small loop. Angenent's lima con example is convex and does not have this sign change of the curvature.

\section{B Variation of Parameters}

The curve is made up of several clearly identifiable parts: the straight pieces in the center, the straight pieces in the outside, the inner circle, the two semi-circles on the left, the outer semi-circle on the right, plus the necessary transitions to make the curve smooth. The question arises, what happens if one changes some of those parameters? In the next few paragraphs, unless said otherwise, we change only one parameter at a time.

\section{The length of the outer straight parts.}

The effect of changing the length of the outer straight part is essentially coming through the change of the length $L$ of the curve, and hence of the average of the curvature. The overlap in the center occurs because the curvature there is initially zero, and adding the average of the curvature to the normal velocity creates the outward movement. Increasing the length $L$ results in reducing $\bar{\kappa}$-compare equation (2.1) - and therefore reduces the force that causes the overlap. The overlap is after all what keeps the inner circle from pulling through the slit, hence a sufficiently large increase of $L$ should cause an avoidance of the singularity. We do not call a loss of embeddedness a singularity. This is the case. For example, is the length of the outer straight parts increased by 1 , then the curve stays regular. For comparison, the original example above has outer straight parts of length about 3 .

\section{The length of the inner straight parts.}

The effect of changing the length of the inner straight part is essentially coming through the change of the size of the overlap of the curve, and the reasoning is then analogous to the one in the previous paragraph. Hence a sufficiently big reduction of the length of the inner straight parts should cause an avoidance of the singularity, and this is the case. For example, is the length of the inner straight parts decreased by $1 / 4$, then the curve stays regular. The original example above has inner straight parts of length about $7 / 4$.

\section{The radius of the inner circle.}

If the radius of the inner circle is increased, the inner circle will move in more slowly and will give more time for the rest of the curve to follow its tendency to pull the overlap back apart. This effect does in fact occur. However, increasing the inner radius does of course also increase the length of the curve, and hence there is a coupling with the effect described two paragraphs above. If one increases the radius from 1, which is the radius of the example, to $5 / 4$, then the curve pulls back apart before the inner circle closes down, and the curve stays regular. Is on the other hand the radius of the inner circle reduced significantly, then it will close down before the overlap at all has time to occur, and the evolution will pull the part coming from the inner circle through the slit, and no singularity will occur. Numerically this happens when the inner radius is $1 / 4$, for example, but does not yet happen when the inner radius is $1 / 2$. 


\section{Other comments.}

It is quite obvious that one can of course change the width of the slit directly and obtain similar effects to the ones described above. The idea of the previous three sections was not to give an exhaustive list of parameter twiddling, but rather to show that the behavior of the evolution is very subtle. As mentioned in the introduction, a curve similar to the example presented here was already numerically investigated in [Mayer and Simonett 2000]. The curve there had an inner radius of $1 / 4$ larger and outer straight parts that were about $1 / 5$ shorter than the corresponding sizes of the example here. The combined effect on the length of the curve is such that the example here is shorter. As outlined in the previous paragraphs both the change of the inner radius and the change of the length tend to remove the singularity, and they did. In fact, the author had no idea at the time of writing of the former paper that with a slight change of parameters a singularity could be forced to occur.

As of this writing the mathematical literature contains essentially two types of results about existence of smooth solutions: short-term existence results for arbitrary curves, and long-term existence results for

convex curves [Gage 1986; Huisken 1987; Escher and Simonett 1998]. Escher and Simonett also show that if the embedded initial curve is close to a circle but not necessarily convex, existence is still guaranteed for all time, and the curve will approach some possibly other circle. According to our conjecture, in contrast, we do not expect that one can show long-term existence for all embedded smooth initial curves, and it is at this stage far from clear which initial curves enjoy a long-term future under the averaged mean curvature flow.

\section{REFERENCES}

[Angenent 1991] S. Angenent, "On the formation of singularities in the curve shortening flow", J. Differential Geom., 33 (1991), 601-633.

[Brakke 1992] Kenneth E. Brakke, "The surface evolver", Experiment. Math., 1:2 (1992), 141-165.

[Escher and Simonett 1998] J. Escher and G. Simonett, "The volume preserving mean curvature flow near spheres", Proc. Amer. Math. Soc., 126:9 (1998), 2789-2796.

[Gage 1986] M. E. Gage, "On an area-preserving evolution equation for plane curves", pp. 51-62. in Nonlinear problems in geometry (Mobile, Ala., 1985), edited by D. M. DeTurck, Contemporary Mathematics 51, Amer. Math. Soc., Providence, 1986.

[Gage and Hamilton 1986] M. E. Gage and R. S. Hamilton, "The heat equation shrinking convex plane curves", J. Differential Geom., 23:1 (1986), 69-96.

[Grayson 1987] M. A. Grayson, "The heat equation shrinks embedded plane curves to round points", $J$. Differential Geom., 26:2 (1987), 285-314.

[Huisken 1987] G. Huisken, "The volume preserving mean curvature flow", J. Reine Angew. Math., 382 (1987), 35-48.

[Mayer 2000] U. F. Mayer, "A numerical scheme for moving boundary problems that are gradient flows for the area functional", Europ. J. Appl. Math., 11:2 (2000), 61-80.

[Mayer and Simonett 2000] U. F. Mayer and G. Simonett, "Self-intersections for the surface diffusion and the volume preserving mean curvature flow", Differ. Integral Equations, 13:7-9 (2000), 1189-1199.

Fachbereich 17 Mathematik, Universität Gesamthochschule Kassel, 34109 Kassel, Germany

(mayer@mathematik.uni-kassel.de)

Received June 12, 2000; accepted in revised form August 24, 2000 